\title{
Targeted mutagenesis of BRANCHED HEAD homoeoalleles causes alterations of wheat spike and root architecture
}

\author{
Hertig C. ${ }^{1 *}$, Marthe C. ${ }^{1}$, Poursarebani N. ${ }^{1}$, Budhagatapalli N. ${ }^{1}$, Hiekel S. ${ }^{1}$, Junker A. ${ }^{1}$, \\ Jacobi A. ${ }^{2}$, Schnurbusch T. ${ }^{1}$, Kumlehn J. ${ }^{1}$ \\ ${ }^{1}$ Leibniz Institute of Plant Genetics and Crop Plant Research (IPK) Gatersleben, Germany \\ ${ }^{2}$ Strube Research GmbH \& Co. KG, Söllingen, Germany \\ * email: hertig@ipk-gatersleben.de
}

The BRANCHED HEAD $(B H)$ gene of the temperate cereal wheat (Triticum aestivum L.) plays a remarkable role in the shaping of spike architecture that itself is a major determinant of the grain number produced per spike and thus of grain yield potential. By expressing specified guide RNAs (gRNA) and Cas9 endonuclease via biolistic DNA transfer to immature embryos followed by plant regeneration, suitable target motifs were addressed within the BRANCHED HEAD $(B H)$ coding sequences present in the three subgenomes of hexaploid wheat. To increase the efficiency of targeted mutagenesis, two regions conserved among the three $B H$ homeoalleles were targeted simultaneously. Mutated target motifs were observed in stably transgenic plants, but, intriguingly, also in transgene-free ones, where transient expression of gRNA and Cas 9 right after DNA transfer must have been sufficient to trigger gene-specific alterations. Target regionspecific PCR amplification and Sanger sequencing identified single, double and triple mutant plants. Additional combinations of loss-of-function homeoalleles were generated by crossing respective single mutant plants. Haploid technology was employed to generate a variety of lines with individual or combined mutant alleles being genetically fixed. A phenotypic evaluation revealed excessive formation of supernumarary spikelets as well as branching of spikes in cases of double and triple homeoallele knockout plants, which was, however, associated with a high rate of floret infertility. Astonishingly, an analysis of some single $b h$ allele knockout plants also revealed alterations in root development. Further efforts are being devoted to the development of allelic variants entailing increased grain numbers per spike while retaining high fertility. 